\title{
La liberté de vendre des clopes...
}

\author{
Samia Hurst \\ Dr med., Institut Ethique Histoire Humanités (iEH2), Faculté de médecine, Genève
}

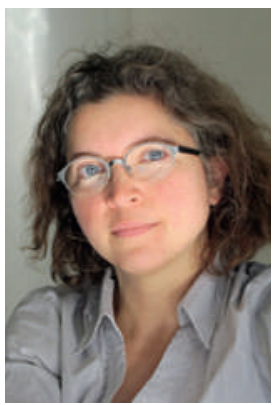

Selon l'Office fédéral de la santé publique, presque 10000 personnes décèdent en Suisse chaque année prématurément en raison du tabagisme. Selon l'Organisation mondiale de la Santé, on peut prévoir sur la base de la consommation actuelle que le tabagisme sera devenu le premier facteur de mortalité à l'échelle mondiale en 2030. Sous l'angle de la médecine, les choses sont depuis longtemps d'une clarté limpide: fumer est dangereux pour la santé et pour la vie, celles des fumeurs et aussi celles de leur entourage. Après près d'un siècle de résistance tenace à la preuve des faits [1], même l'industrie du tabac a dû admettre ces conclusions. Dans ce contexte, les discussions qui entourent la loi sur les produits du tabac ont une importance évidente en termes de santé.

Evidemment, le tabagisme a aussi une importance économique en Suisse. Ce sont des emplois, des impôts, des financements industriels. On doit donc s'attendre à ce que toute tentative de légiférer sur la prévention du tabagisme se heurte, forcément, à ces intérêts. A l'échelle du monde, cet affrontement peut devenir impressionnant. Depuis quelques temps, lorsque des nations veulent protéger leur population par des mesures visant à diminuer la consommation de cigarettes, l'industrie du tabac semble avoir pris l'habitude

\section{Un affrontement se joue ici entre deux lignes de force de nos sociétés.}

de les traîner devant l'équivalent commercial de tribunaux internationaux. L'atteinte à la perspective de faire du profit est semble-t-il désormais punissable. Un affrontement se joue ici entre deux lignes de force de nos sociétés: la promotion de la santé et la promotion du profit commercial.

Rien de surprenant, donc, lorsque le projet de loi sur les produits du tabac se heurte, en Suisse aussi, à des réticences. Comme la confrontation entre la santé et le commerce est inconfortable, cela dit, on lui préfère un enjeu plus confortable. Si l'on a des doutes sur le bien- fondé du projet de loi sur les produits du tabac, ce n'est pas une question d'argent. Non, c'est parce que l'on est attaché à la liberté. C'est très important, la liberté. Ceux qui sont contre peuvent-ils lever la main, s'il vous plaît? Personne? Vous voyez bien...

\section{La liberté des entreprises est ici en concurrence avec celle des personnes.}

Il faut cependant comprendre que ce n'est pas la liberté du fumeur qui est ici en cause. La plupart des fumeurs sont devenus dépendants de la cigarette pendant leur adolescence. Comme exemple du libre choix d'adultes consentants, il y a mieux. Malgré cela, pas question d'interdire la cigarette: chacun restera libre de fumer. Non, la liberté que l'on défend ici c'est celle de l'industrie du tabac. C'est la liberté de faire la promotion d'un produit dangereux et addictif, dont on tire un profit. Il faut aussi comprendre que cette liberté des entreprises est ici en concurrence avec celle des personnes. Face aux produits du tabac, nous devons faire un choix: donner plus de liberté au vendeur pour promouvoir ses produits, c'est limiter les possibilités des citoyens d'être libres de la cigarette. Cette conclusion n'est pas théorique. Faire la promotion des produits du tabac a pour but explicite d'en augmenter la consommation. Interdire la publicité diminue la consommation [2] et avec elle le nombre de personnes dépendantes qui préféreraient arrêter [3]. Alors, à la liberté de qui sommesnous attachés? C'est là, au fond, qu'est la question.

\section{Références}

1 Brandt A. The Cigarette Century: the Rise, Fall, and Deadly Per sistence of the Product that Defined America. New York: Basic Books; 2009.

2 Saffer $\mathrm{H}$, Chaloupka F. The effect of tobacco advertising bans on tobacco consumption. Journal of health economics. 2000;19(6):1117-37.

3 Siahpush M, Yong HH, Borland R, et al. Smokers with financial stress are more likely to want to quit but less likely to try or succeed: findings from the International Tobacco Control (ITC) Four Country Survey. Addiction. 2009;104(8):1382-90. 